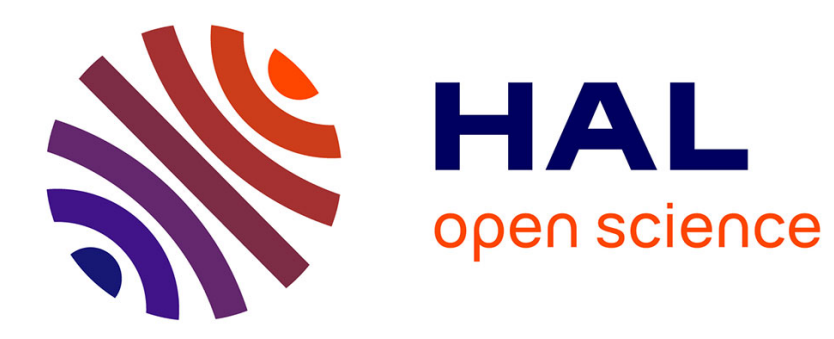

\title{
Mesure de rotations Faraday dans un laser anisotrope
}

\author{
A. Le Floch, G. Stephan
}

\section{To cite this version:}

A. Le Floch, G. Stephan. Mesure de rotations Faraday dans un laser anisotrope. Revue de Physique Appliquée, 1975, 10 (1), pp.1-6. 10.1051/rphysap:019750010010100 . jpa-00243874

\section{HAL Id: jpa-00243874 https://hal.science/jpa-00243874}

Submitted on 1 Jan 1975

HAL is a multi-disciplinary open access archive for the deposit and dissemination of scientific research documents, whether they are published or not. The documents may come from teaching and research institutions in France or abroad, or from public or private research centers.
L'archive ouverte pluridisciplinaire HAL, est destinée au dépôt et à la diffusion de documents scientifiques de niveau recherche, publiés ou non, émanant des établissements d'enseignement et de recherche français ou étrangers, des laboratoires publics ou privés. 


\title{
REVUE DE PHYSIQUE APPLIQUÉE
}

Supplément au « Journal de Physique *

Classification

Physics Abstracts

$2.630-8.500$

\section{MESURE DE ROTATIONS FARADAY DANS UN LASER ANISOTROPE}

\author{
A. LE FLOCH et G. STEPHAN
}

Laboratoire d'Electronique quantique

Faculté des Sciences, 35-Rennes, France

(Reçu le 5 septembre 1973, révisé le 18 février 1974)

\begin{abstract}
Résumé. - La théorie spatiale vectorielle basée sur la condition de résonance pour représenter un laser anisotrope offre une méthode de mesure de rotations Faraday. La rotation Faraday simple passage est compensée par une anisotropie de pertes, connue, du type $x-y$. La rotation, mesurable, du vecteur propre à une extrémité de la cavité, est beaucoup plus importante. On définit ainsi les pouvoirs multiplicateurs statique et dynamique de la cavité. Cette méthode permet de mesurer des rotations Faraday de $2 \times 10^{-6}$ degré dans l'infrarouge.

Abstract. - The spatial vectorial theory based on the resonance condition for representing an anisotropic laser, provides a method for measuring Faraday rotations. The Faraday rotation per path is compensated by means of a known, x-y-type loss anisotropy. The measured rotation of the eigenvector at one end of the laser is very large. We then define static and dynamic gains for the cavity. This method enables us to measure Faraday rotations in the infrared as small as $2 \times 10^{-6}$ degree.
\end{abstract}

1. Introduction. - Les mesures de rotations Faraday et de dichroïsme circulaire, usuellement effectuées $[1,2]$ pour obtenir des renseignements spectroscopiques parfois inaccessibles par d'autres techniques, se font par simple passage d'un faisceau de lumière à travers la substance étudiée. Pour les rotations Faraday faibles, certains auteurs comme Rosenberg et al. [3], ou Clair [4], ont cherché à utiliser le caractère nonréciproque de cette rotation en réalisant des appareillages à multi-passages, comme les cavités Fabry-Perot isotropes par exemple. Dans ces expériences, le résonateur utilisé est passif et nécessite l'utilisation d'une source lumineuse extérieure, ce qui crée un problème d'accord entre la fréquence de la source lumineuse et les fréquences de résonance du Fabry-Perot. L'ensemble donne pour un signal d'entrée polarisé linéairement, une résultante elliptique, dont on mesure la rotation du grand axe, ce qui limite la précision de la méthode.

Doyle et White [5] ainsi que Chen et al. [6] ont proposé des méthodes de mesure de rotation Faraday dans des résonateurs actifs anisotropes, basées respectivement sur des variations de fréquences et d'intensité, dépendant de la rotation Faraday simple passage.
Nous avons montré que l'analyse correcte de chacun de ces types de résonateur anisotrope, nécessitait une étude particulière, la position de chaque anisotropie dans la cavité étant essentielle [7]. Nous présentons dans cet article une méthode portant sur l'observation de la polarisation de sortie du laser. Pour les lasers comportant des anisotropies de pertes du type x-y $\left({ }^{1}\right)$ (fenêtre de Brewster par exemple [8]), nous avons notamment mis en évidence l'existence en un point donné, de deux vecteurs lumineux distincts correspondant chacun à un sens de propagation dans la cavité [9]. La polarisation de sortie du laser à une extrémité donnée, fixée par le vecteur propre oscillant en ce point de la cavité peut subir des rotations énormes par rapport à la rotation Faraday en simple passage. Il suffira de mesurer la rotation de la polarisation de sortie du laser pour pouvoir en déduire la rotation Faraday que l'on cherche à mesurer.

Nous allons d'abord rappeler la théorie vectorielle

(1) Dans notre cas de cavité nous distinguerons deux types d'anisotropies :

- les anisotropies de pertes du type xy (linéaires),

- les anisotropies de phases circulaires (ici rotation Faraday). 
pour le type de cavité utilisée et définir ce que nous avons appelé le vecteur de Lamb qui est utilisé pour la mesure. Ceci permettra d'établir la relation entre la rotation Faraday à mesurer et la rotation du vecteur de Lamb. Il en résulte en particulier, l'apparition d'un pouvoir multiplicateur de la cavité qui fait l'intérêt de notre méthode. Dans un second paragraphe nous décrirons la réalisation expérimentale et nous indiquerons les possibilités de la méthode, avec les variantes possibles.

2. Rotation du vecteur de Lamb. Pouvoirs multiplicateurs de la cavité résonnante. -2.1 RoTATION DU VECTEUR DE LAMB. - Un oscillateur quantique comportant des effets non-réciproques a été étudié dans la référence [7]. Il était constitué (Fig. 1a) par une colonne gazeuse active soumise à un champ magnétique axial qui crée la rotation Faraday, et par deux miroirs anisotropes $R_{1}$ et $R_{2}$. Le laser monomode, était accordé au centre de la raie. Le but de l'étude était de montrer que la théorie actuellement utilisée [10] pour décrire les lasers n'est plus correcte avec ce modèle. En effet, le vecteur champ électrique n'a pas la même direction tout au long du laser. Seule une théorie spatiale vectorielle, utilisant la méthode de la condition de résonance peut décrire correctement le champ électromagnétique dans la cavité. Pour un tel laser, la polarisation initiale, linéaire en champ nul, reste linéaire, mais subit une rotation lorsqu'on augmente le champ magnétique appliqué (soit la rotation Faraday) jusqu'à une valeur $H_{\mathrm{c}}$ (champ critique pour lequel la polarisation se décompose en deux vibrations elliptiques). C'est cette région, la région de blocage qui va nous intéresser ici.

Considérons la cavité laser représentée par la figure $1 b$. Elle comprend :

- un milieu actif assurant l'oscillation,

- une anisotropie du type $x-y$ constituée par une lame de silice (le plan d'incidence définit l'axe de moindres pertes $O y$ ),

- un rotateur Faraday (cristal de YIG),

- deux miroirs isotropes.

Ceci est un cas particulier du modèle général de la figure $1 a$, où la rotation Faraday est assurée par un cristal. Sur la figure $1 b$, nous avons également représenté les vecteurs en différents points de la cavité. En particulier il faut noter que la traversée de la lame de silice (la transformation de $\mathbf{E}_{1}$ en $T \mathbf{E}_{1}$ ) s'accompagne d'une rotation du plan de polarisation et rapproche la vibration du plan d'incidence [11].

La distribution vectorielle du champ pour un aller et retour dans la cavité est représentée par la figure $1 c\left({ }^{2}\right)$. $\theta$ est l'angle mesurable, à la sortie du

(2) Nous remercions M. Dumont du Laboratoire de Spectroscopie Hertzienne de l'ENS pour nous avoir suggéré ce type de diagramme qui permet de représenter les variations d'orientations du champ électromagnétique pour un aller-retour dans la cavité laser.

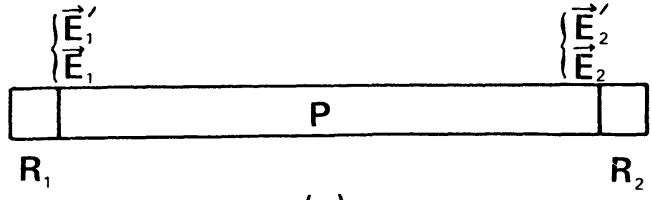

(a)

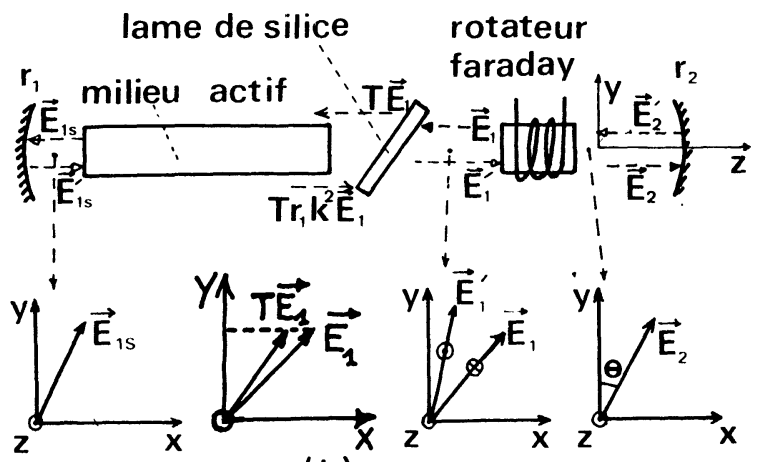

(b)

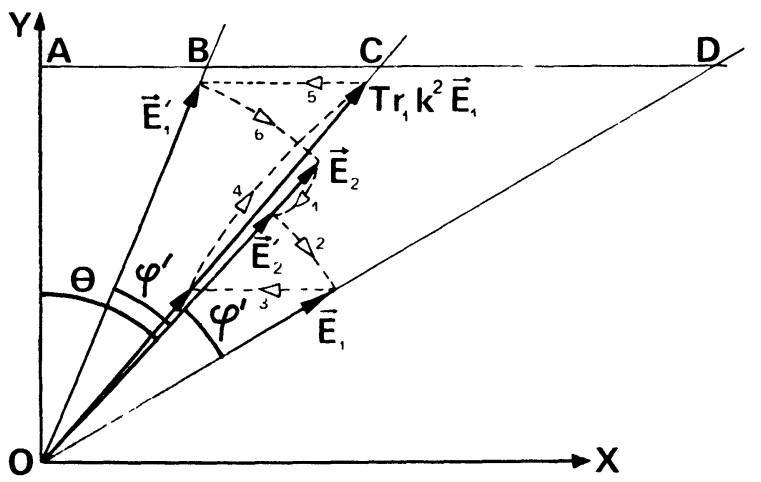

(C)

FIG. 1. - Schéma du résonateur. 1a) modèle théorique ; 1b) modèle expérimental et diagrammes vectoriels en différents points du résonateur ; 1 c) distribution vectorielle du champ pour un aller un et retour dans la cavité :1) pertes sur $\left.r_{2} ; 2\right)$ rotation Faraday simple passage vers les $z$ négatifs ; 3 ) passage de $l$ vers les $z$ négatifs ; 4) amplification due au milieu actif en double passage ; 5) passage de $l$ vers les $z$ positifs ; 6) rotation Faraday simple passage vers les $z$ positifs. Pour simplifier le dessin, nous avons omis les pertes de la polarisation selon $O y$ à la traversée de $l$ (intégrées dans $r_{1}$ par exemple). Nous n'avons tenu compte que du rapport de pertes entre les polarisations selon $\mathbf{O x}$ et $\mathbf{O y}$ (supposé égal ici à $\frac{1}{2}: \frac{A B}{A C}=\frac{A C}{A D}=\frac{1}{2}$ ). On peut noter que la relation entre la rotation Faraday $\varphi^{\prime}$ et la rotation du vecteur de sortie, soit $\theta$, peut se retrouver géométriquement à partir de cette figure. On a :

$\frac{\sin 2 \theta}{\sin 2 \varphi^{\prime}}=\frac{\operatorname{tg}\left(\theta+\varphi^{\prime}\right)+\operatorname{tg}\left(\theta-\varphi^{\prime}\right)}{\operatorname{tg}\left(\theta+\varphi^{\prime}\right)-\operatorname{tg}\left(\theta-\varphi^{\prime}\right)}=$

$$
=\frac{A D+A B}{A D-A B}=\frac{T+1 / T}{1 / T-T}=\frac{1}{\varepsilon_{1}}
$$

où $T=\frac{T x}{T y}$ et $\varepsilon_{1}=\frac{1-T^{2}}{1+T^{2}}$. D'où $\sin 2 \theta=1 / \varepsilon_{1} \sin 2 \varphi^{\prime}$.

laser, $\varphi^{\prime}$ est la rotation Faraday simple passage $\left(\varphi^{\prime}=\widehat{\mathrm{BOC}}\right)$. On part par exemple du champ $\mathbf{E}_{2}^{\prime}$ incliné d'un angle $\theta$ sur $O y$. Après traversée du rotateur, la polarisation devient $\mathbf{E}_{1}$. Le milieu de pertes anisotropes transforme $\mathbf{E}_{1}$ en $T \mathbf{E}_{1}$ donnant ainsi une 
rotation à la polarisation. Remarquons que cette rotation est non réciproque, puisqu'elle tend toujours à rapprocher la vibration optique de l'axe de plus faibles pertes $O y$. Le diagramme $1 c$, qui exprime qu'après un aller-retour dans la cavité, le champ se retrouve identique à lui-même, se comprend donc physiquement d'une manière très simple : la rotation Faraday à chaque passage est compensée par une rotation en sens inverse due à l'anisotropie de pertes. La relation entre $\varphi^{\prime}$ et $\theta$ que nous donnons plus bas (le pouvoir multiplicateur) n'est donc pas due à un effet multipassage mais à un effet compensateur de l'anisotropie pour chaque aller-retour dans la cavité.

Il reste à exprimer la relation entre la rotation Faraday $\varphi^{\prime}$ que l'on cherche à déterminer et la rotation du vecteur propre en un point de la cavité que l'on pourra mesurer (soit $\theta$ dans le cas présent). Nous allons refaire ici les calculs permettant de déduire les polarisations du champ du laser dans le cas précis qui nous préoccupe où la rotation Faraday n'est pas due à la colonne active même du laser, mais à un rotateur Faraday rajouté dans la cavité (Fig. 1b). Dans la base des ondes de polarisations circulaires le rotateur Faraday sera représenté par une matrice de Jones :

$$
F=\left[\begin{array}{ll}
\mathrm{e}^{i \varphi / 4} & 0 \\
0 & \mathrm{e}^{-i \varphi / 4}
\end{array}\right]
$$

telle que $\mathbf{E}_{2}=F \mathbf{E}_{1}^{\prime}$ où $\varphi / 4=\varphi^{\prime}$ est la rotation Faraday en simple passage dans le cristal de YIG. Désignons par $r_{1}$ et $r_{2}$ le pouvoir réflecteur des miroirs isotropes se trouvant aux extrémités 1 et 2 du laser, $T$. la matrice décrivant la transmission à travers la lame de silice, $k$ le coefficient d'amplification saturée de la vapeur. La condition de résonance impose que pour un aller et retour le champ électrique se retrouve identique à lui-même. Appliquée à l'extrémité 2 (vecteur $\mathbf{E}_{2}$ incident sur $r_{2}$ ) cette condition s'écrit :

$$
\text { F.T.k. } r_{1} \text { k.T.F. } r_{2} \mathbf{E}_{2}=\mathbf{E}_{2} \text {. }
$$

En posant :

$$
R_{1}=T^{2} r_{1} ; \quad R_{2}=r_{2} ; \quad P=k . F .
$$

On obtient :

$$
P R_{1} P R_{2} \mathrm{E}_{2}=\mathrm{E}_{2}
$$

ce qui montre que l'on est ramené au cas de la figure $1 a$ où les anisotropies étaient incluses dans les miroirs.

Dans la base circulaire, la matrice $R_{1}=r_{1} T^{2}$ s'écrira sous la forme :

$$
R_{1}=\left[\begin{array}{rr}
a & -b \\
-b & a
\end{array}\right]
$$

avec

$$
a=\frac{R_{x}+R_{y}}{2} ; \quad b=\frac{R_{x}-R_{y}}{2}
$$

$R_{x}=T_{x}^{2} ; R_{y}=T_{y}^{2}$ où $T_{x}$ et $T_{y}$ sont respectivement les transmissions de la lame de silice dans les plans perpendiculaire et parallèle au plan d'incidence.
Elles sont fixées par les formules de Fresnel :

$$
\left\{\begin{array}{l}
T_{x}=\frac{\sin 2 i \sin 2 r}{\sin ^{2}(i+r)} \\
T_{y}=\frac{\sin 2 i \cdot \sin 2 r}{\sin ^{2}(i+r) \cdot \cos ^{2}(i-r)}
\end{array}\right.
$$

où $i$ est l'angle d'incidence, $r$ l'angle de réfraction défini par sin $i=[n \cdot \sin r$. Rappelons que dans le cas général d'une cavité terminée par deux miroirs anisotropes $R_{1}$ et $R_{2}$ de la figure $1 a$, les vecteurs $\mathbf{E}_{z}$ et $\mathbf{E}_{z}^{\prime}$ représentant les ondes se propageant en sens inverse au point $z$ ne sont pas confondus. C'est le cas notamment pour $\mathbf{E}_{\mathbf{1}}$ et $\mathbf{E}_{1}^{\prime}$ dans le cas présent de la figure $1 b$. Ceci résulte du caractère non réciproque de l'effet Faraday et du passage à travers la lame de silice.

Toutefois, nous avons montré qu'il existe un point $z$ pour lequel les deux vecteurs à l'aller et au retour sont colinéaires: c'est ce que nous avons appelé le vecteur de Lamb, dont la rotation maximale est de $\pi / 4$. Le vecteur de Lamb se trouve être le vecteur $\mathbf{E}_{2}$ [12]. Pour une rotation Faraday $\varphi^{\prime}$ le vecteur $\mathbf{E}_{2}$ tourne d'un angle tel que :

$$
\sin 2 \theta=\frac{1}{\varepsilon_{1}} \sin \frac{\varphi}{2}=\frac{1}{\varepsilon_{1}} \sin 2 \varphi^{\prime}
$$

où

$$
\varepsilon_{1}=\frac{T_{x}^{2}-T_{y}^{2}}{T_{x}^{2}+T_{\nu}^{2}} .
$$

Cette relation, que l'on peut retrouver géométriquement dans le cas de la figure $1 c$ (voir la légende de cette figure), permet donc, en mesurant la rotation du vecteur $\mathbf{E}_{2}$ de sortie, soit $\theta$, d'évaluer la rotation Faraday simple passage.

2.2 Pouvoirs multiplicateurs. - Pour diverses anisotropies données, la rotation du vecteur propre en fonction de la rotation Faraday simple passage est portée sur la figure 2. Nous remarquons que la

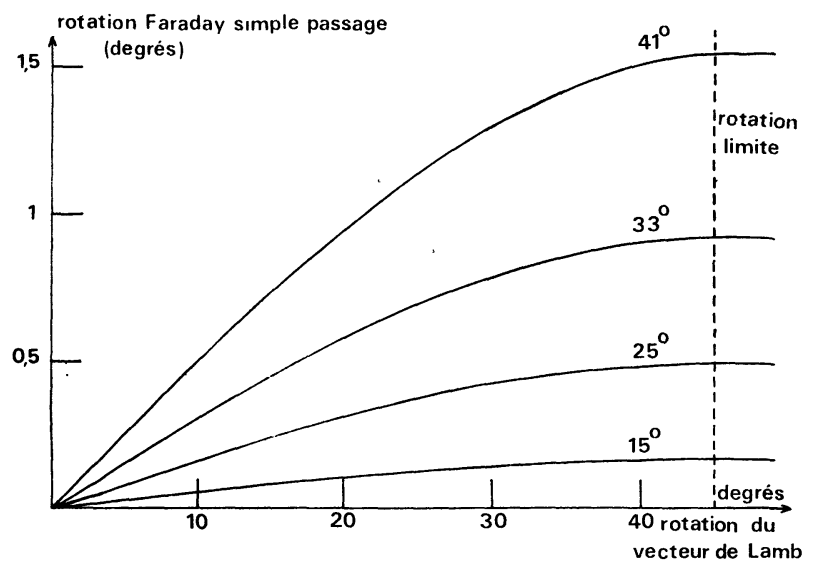

FIG. 2. - Rotation Faraday simple passage en fonction de l'azimut du vecteur de Lamb $\mathbf{E}_{2}$ pour diverses valeurs de l'anisotropie (soit pour diverses inclinaisons de la lame de silice, ici $41^{\circ}, 33^{\circ}, 25^{\circ}, 1^{\circ}$ ). 
rotation maximale du vecteur de Lamb est de $45^{\circ}$, quelle que soit la valeur de l'anisotropie. L'examen de ces courbes laisse prévoir deux possibilités pour la mesure de rotation Faraday:

2.2.1 Méthode statique, utilisant le pouvoir multiplicateur statique. $-\mathrm{Si}$ on pose

$$
\theta=\mathcal{M} \varphi^{\prime}
$$

on voit que $\mathcal{H}$ sera le pouvoir multiplicateur de la cavité, qui dépendra de la valeur de $\varepsilon_{1}$ et de la rotation du vecteur de Lamb. Pour une anisotropie fixée, on mesure la rotation du vecteur de Lamb. A partir de la courbe correspondant à l'anisotropie, on déduit la rotation Faraday. Ici on utilise le pouvoir multiplicateur statique défini par les relations (7) et (8), et que l'on a représenté sur la figure 3 . Cette méthode convient pour des rotations Faraday relativement fortes (quelques dizièmes de degrés).

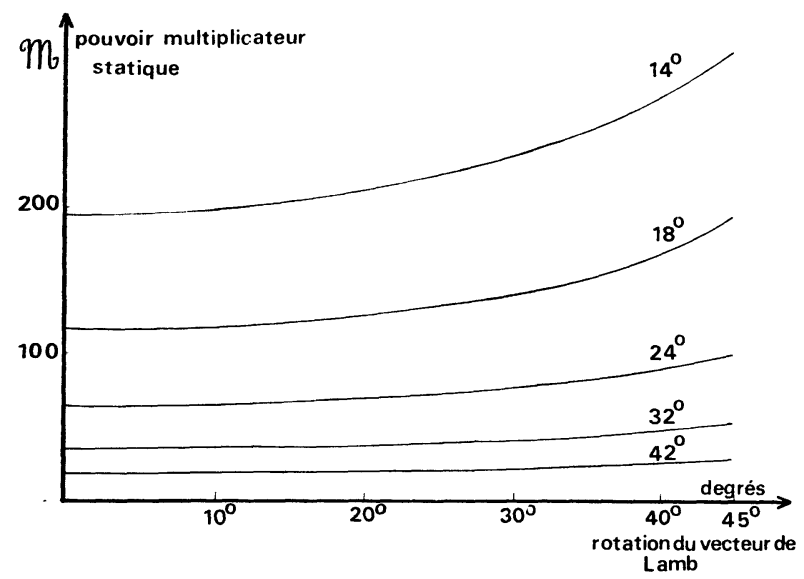

Fig. 3. - Courbes théoriques donnant le pouvoir multiplicateur statique pour diverses valeurs de l'anisotropie.

2.2.2 Méthode dynamique; pouvoir multiplicateur dynamique. - On peut remarquer qu'à partir de la forme des courbes de la figure 2 , on peut utiliser une méthode dynamique utilisant la pente de ces courbes. On peut d'ailleurs définir un pouvoir multiplicateur dynamique $\mathcal{H}^{\prime}$ par la relation déduite de la relation (7).

$$
\mathcal{H}^{\prime}=\frac{\mathrm{d} \theta}{\mathrm{d} \varphi^{\prime}}=\frac{1}{\varepsilon_{1}} \frac{\cos 2 \varphi^{\prime}}{\cos 2 \theta} .
$$

Les courbes représentant $\mathcal{M}^{\prime}$ sont portées sur la figure 4. On constate que $\mathcal{H}^{\prime}$ tend vers l'infini lorsque $\theta$ tend vers $45^{\circ}$. Cette méthode pourra s'utiliser en superposant une petite composante alternative $H_{1} \cos \omega t$ au champ $H_{0}$ qui fixe le point de fonctionnement du montage. Ceci permettra des mesures beaucoup plus fines.

3. Montage expérimental. Résultats. - 3.1 MéTHODE STATIQUE. - La méthode de mesure est classique et représentée sur la figure 5. Le laser ici monomode, oscillant sur la raie $3,39 \mu$; a déjà été décrit dans la

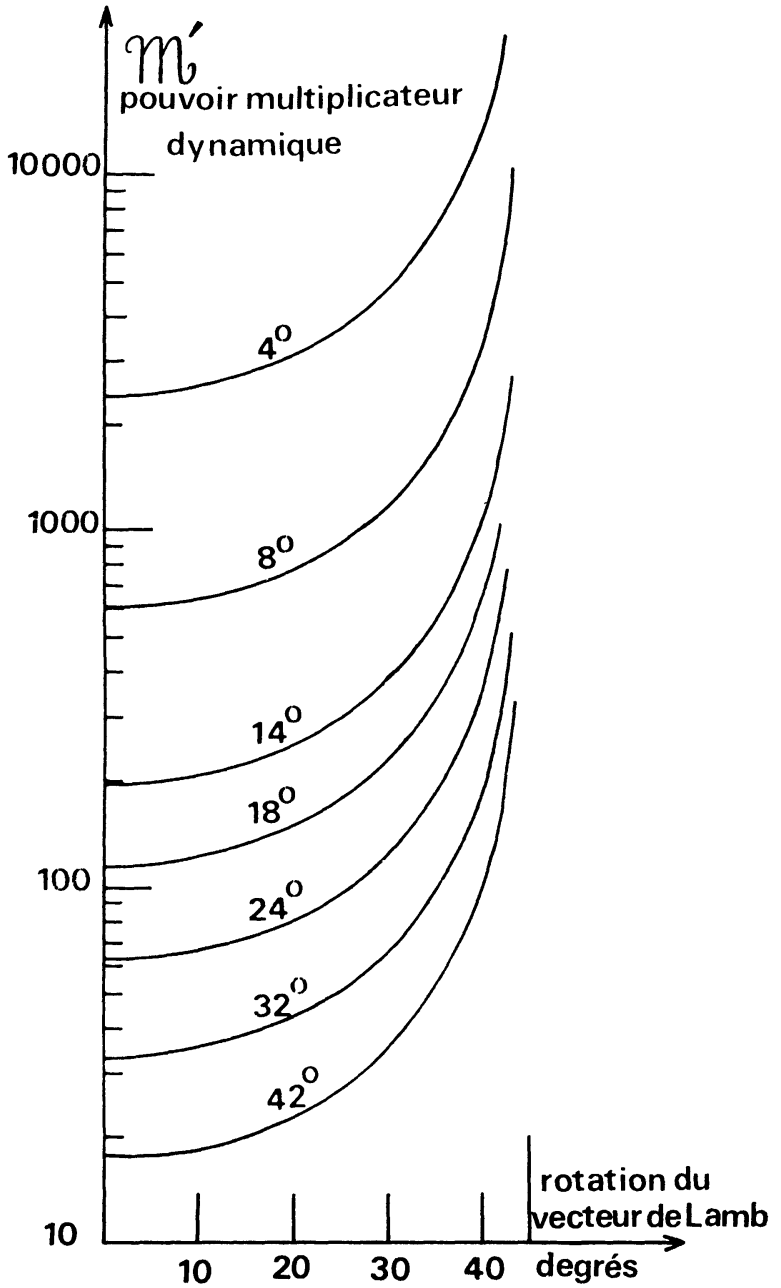

FIG. 4. - Courbes théoriques donnant le pouvoir multiplicateur dynamique pour diverses valeurs de l'anisotropie.

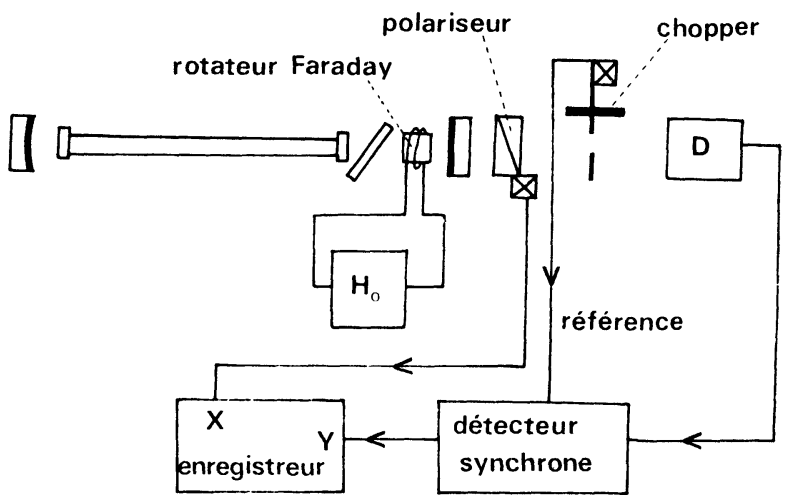

FIG. 5. - Montage 1 utilisant la méthode statique.

référence 9. L'anisotropie est constituée par une lame de silice $(n=1,41)$ inclinable d'un angle $i$ par rapport à la direction du faisceau laser. Le cristal Faraday est un cristal de YIG taillé (111). L'analyse de la lumière de sortie se fait grâce à un polariseur tournant en calcite. Le flux lumineux est haché et la mesure se fait par détection synchrone. Un enregistreur $X-Y$ permet de lire directement la rotation $\theta$ correspondant 
à un champ donné. Un exemple de courbes obtenues est représenté sur la figure 6 . Dans cette méthode, la précision sur la mesure de la rotation Faraday est donnée par la formule :

$$
\frac{\Delta \varphi}{\varphi}=\frac{\Delta \mathcal{N}}{\mathscr{M}}+\frac{\Delta \theta}{\theta} .
$$

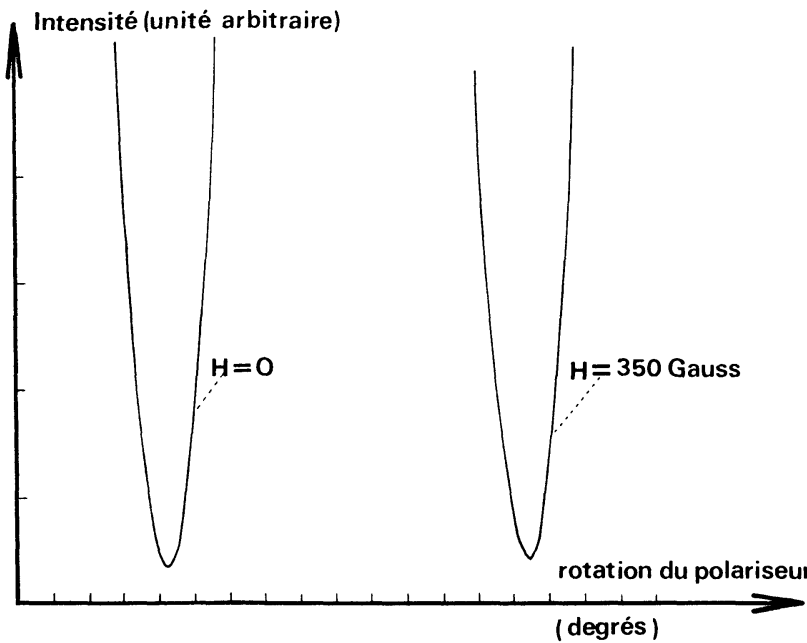

Fig. 6. - Résultats expérimentaux obtenus pour 2 valeurs du champ magnétique.

Si l'on considère par exemple la position de la lame connue à $0,5^{\prime}$ près, ceci conduit à $\Delta \mathcal{H} / \mathcal{H} \simeq 8 \times 10^{-4}$, pour une inclinaison $i$ de la lame et une rotation du vecteur de $\mathrm{Lamb}$ de $20^{\circ}$. La précision sur la mesure de $\theta$ dans la méthode statique, peut atteindre 1 ", si l'on utilise une modulation de la polarisation comme l'a montré Nomarski $[13]\left( \pm 1^{\prime \prime}\right)$. On a alors

$$
\frac{\Delta \theta}{\theta} \simeq 2 \times 10^{-5} \text { et } \frac{\Delta \varphi}{\varphi} \simeq 8 \times 10^{-4} .
$$

La figure 7 représente la variation $\Delta \mathcal{H} / \mathcal{H}$ en fonction de l'angle de la lame $i$ pour 4 valeurs de l'incertitude di sur $i$. Si l'on veut avoir $\Delta \mathcal{H} / \mathcal{H} \simeq \Delta \theta / \theta$ on voit que $i$ doit être de l'ordre de $30^{\circ}$ pour $\mathrm{d} i=1^{\prime \prime}$. Dans le cas présent, on a simplement utilisé une méthode directe de mesure de $\theta$, comme l'indique la figure 6 . Pour un cristal ferrimagnétique tel que le YIG, on ne peut définir une constante de Verdet $V$, dans le sens usuel [14]. Cependant, pour la partie linéaire de la courbe de la rotation Faraday en fonction de $\boldsymbol{H}$ qui nous intéresse, nous obtenons $V=0,075 \% \mathrm{~cm} . \mathrm{G}$.

3.2 Méthode DynAMique. - Le schéma utilisé pour cette méthode est représenté par la figure 8 . La détection est comparable à celle utilisée par Badoz [1]. Ici le polariseur est fixe et éteint le faisceau pour la valeur $H_{0}$ du champ statique. On superpose à $H_{0}$, deux modulations $H_{1} \cos \omega_{1} t$ et $H_{2} \cos \omega_{2} t$ de fréquences nettement différentes $\left(\omega_{1}=2 \pi 40\right.$ et $\omega_{2}=2 \pi$ ), auxquelles correspondront des rotations $\alpha_{1}=\alpha_{1 \mathrm{~m}} \cos \omega_{1} t$ et $\alpha_{2}=\alpha_{2 \mathrm{~m}} \cos \omega_{2} t$ du plan de

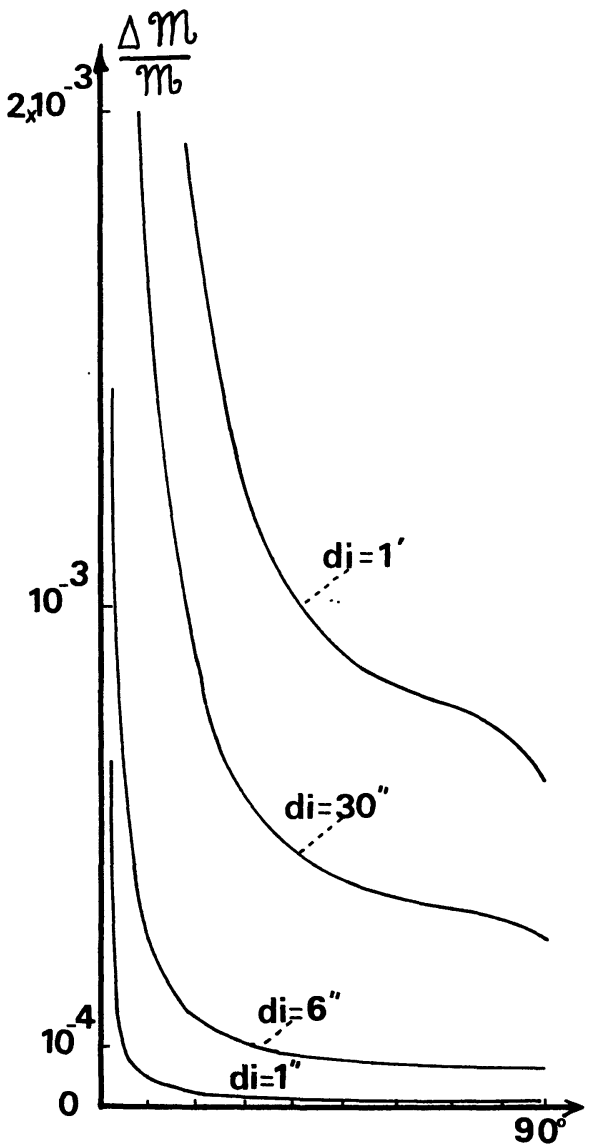

FIG. 7. $-\dot{\Delta} \mathcal{M} / \mathcal{M}$ en fonction de l'angle d'incidence $i$ sur la lame.

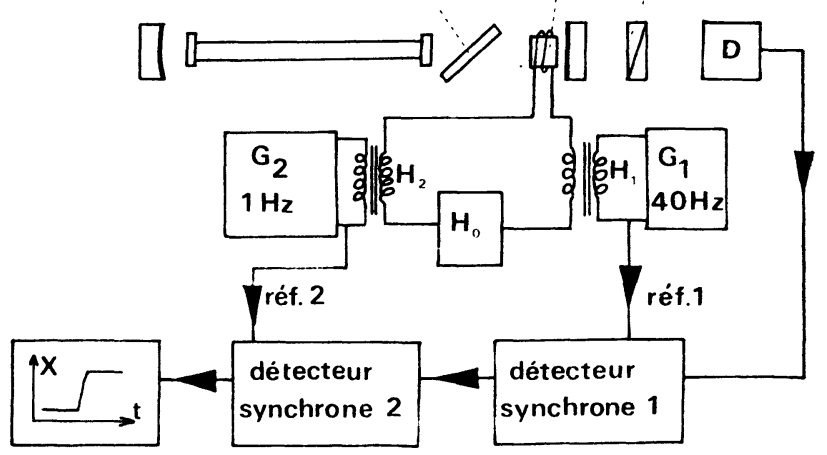

Fig. 8. - Montage 2 utilisant la méthode dynamique.

polarisation. Si $I_{0}$ est l'intensité du faisceau arrivant sur le polariseur, l'intensité après la traversée s'écrit :

$$
I=I_{0} \sin ^{2}\left(\alpha_{1}+\alpha_{2}+\alpha_{0}\right)+I_{\mathrm{p}}+I_{\mathrm{d}}
$$

$\alpha_{0}$ correspond au décalage entre la ligne neutre du polariseur et le vecteur lumineux lorsque le laser est soumis à $H_{0}$. Ce terme $\alpha_{0}$ contient les dérives lentes possibles du plan de polarisation due aux instabilités du montage. $I_{\mathrm{p}}$ est l'intensité lumineuse parasite et $I_{\mathrm{d}}$ l'intensité équivalente au bruit propre du détecteur. 
Comme les angles $\alpha_{0}, \alpha_{1}, \alpha_{2}$ sont faibles le signal $I$ peut s'écrire :

$$
\begin{aligned}
I=I_{0}\left(\alpha_{\mathrm{m} 1}^{2} \cos ^{2} \omega_{1} t+\alpha_{\mathrm{m} 2}^{2} \cos ^{2} \omega_{2} t+\alpha_{0}^{2}+\right. \\
+2 \alpha_{0} \alpha_{\mathrm{m} 1} \cos \omega_{1} t+2 \alpha_{0} \alpha_{\mathrm{m} 2} \cos \omega_{2} t \\
\left.+2 \alpha_{\mathrm{m} 1} a_{\mathrm{m} 2} \cos \omega_{1} t \cos \omega_{2} t\right)+I_{\mathrm{p}}+I_{\mathrm{d}}
\end{aligned}
$$

Le premier détecteur synchrone, accordé sur $\omega_{1}$ détectera le signal :

$$
s_{1}=2 I_{0} \alpha_{\mathrm{m} 1} \cos \omega_{1} t\left[\alpha_{0}+\alpha_{\mathrm{m} 2} \cos \omega_{2} t\right] .
$$

Le premier terme est un terme lié à $\alpha_{0}$, tenant compte des dérives lentes. Si la constante de temps de la détection est faible (ici $0,3 \mathrm{~s}$ ) à la sortie on aura une modulation due au seul terme :

$$
I_{0} 2 \alpha_{\mathrm{m} 1} \alpha_{\mathrm{m} 2} \cos \omega_{1} t \cos \omega_{2} t .
$$

Le second détecteur synchrone centré sur $\omega_{2}$ ne détecte que ce terme (les dérives sont éliminées). Par conséquent, le signal final détecté est proportionnel à $\alpha_{\mathrm{m} 2}$. Il suffira de faire varier l'amplitude de la modulation à $1 \mathrm{~Hz}$ pour mesurer la sensibilité du montage, la méthode statique permettant un étalonnage en valeur absolue. La constante de temps du second détecteur synchrone est de $100 \mathrm{~s}$. Le bruit provient essentiellement du détecteur infrarouge. Les résultats présents (Fig. 9) permettent d'apprécier $2 \times 10^{-6}$ degré, ce qui améliore par un facteur 100 la sensibilité obtenue par les méthodes usuelles. (En effet l'incertitude relative sur cette mesure est la même que celle portant sur $V$ ). Ceci correspond dans cet exemple (lame à $20^{\circ}$ ) au gain dû au pouvoir multiplicateur dynamique. Les courbes théoriques de la figure 4 laissent prévoir que dans le visible où l'on dispose de meilleurs détecteurs il doit être possible d'améliorer beaucoup la précision de la mesure. Il serait possible de n'utiliser qu'une seule détection synchrone à la fréquence double (détection quadratique). Toutefois la sensibilité de ce montage serait plus faible que celle d'une détection linéaire.

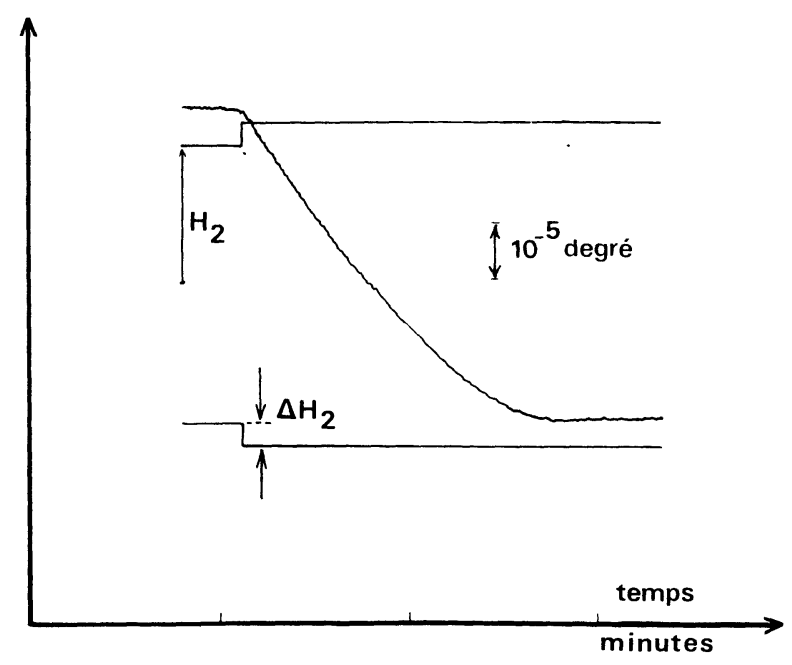

Fig. 9. - Résultats expérimentaux obtenus par la méthode dynamique. On enregistre en même temps la courbe enveloppe du champ $h_{2}=H_{2} \cos \omega_{2} t$ dont on modifie l'amplitude de $\Delta H_{2}$ et la variation correspondante du signal détecté.

4. Conclusion. - L'analyse vectorielle de la polarisation de la lumière dans un laser Zeeman comportant une anisotropie du type $x-y$ peut se faire par la méthode de la condition de résonance. On trouve alors que la polarisation de la lumière à la sortie du laser peut être linéaire. Ceci offre une nouvelle méthode de mesure de rotation Faraday. L'expérience effectuée sur la raie 3,39 $\mu$ du néon, permet de mesurer des rotations très faibles $\left(2 \times 10^{-6}\right.$ degré $)$, et permet une sensibilité d'environ 100 fois supérieure à celle des montages usuels. Cette sensibilité peut être notablement augmentée, le pouvoir multiplicateur théorique pouvant devenir infini, notamment dans le domaine visible où l'on dispose de détecteurs à meilleures performances. Les raies infrarouges telles que 3,39 $\mu$ peuvent être utilisées à mesurer la rotation Faraday dans les semi-conducteurs, (surtout les rotations faibles) pour en déduire la masse effective des porteurs libres, ou pour étudier les transitions interbandes [15].

\section{Références}

[1] Badoz, B. Briat and Billardon, M., Jap.J. Appl. Phys. 4 (1965).

[2] Billardon, M., Rivoal, J. C. and Badoz, J., Revue Phys. Appl. 4 (1969) $\mathrm{n}^{\circ} 3$.

[3] Rosenberg, R., Rubinsten, C. B. and Herriott, D. R., Appl. Optics, 3 (1964) 1079.

[4] Clair, J. J., Optica Acta, 118 (1971) 47.

[5] Doyle, W. M. and White, M B., J. Opt. Soc. Am. 55 (1965) 1221.

[6] DI CHEN et al., IEEE Journal of Quant. Elect. QE 2 (1966) et QE 6 (1970).

[7] Le Floch, A. and Le Naour, R., Phys. Rev. A. 4 )1971) 290 et la référence 4 de cet article.
[8] Guion, W. G. and Craig, J. P., Proc. IEEE, 56 (1968) 1624.

[9] Le Floch, A. and Stephan, G., Phys. Rev. A, 6 (1972) 845 et C. R. Hebd. Séan. Acad. Sci. 274 (1972) 491.

[10] Lamb, W. E., Phys. Rev. 234 (1964) 1429.

[11] Bruhat, G., Cours d'optique (Masson) 1965 § 206, 394.

[12] Ici $\mathbf{E}_{2}$ est le vecteur de Lamb puisque dans le cadre du modèle général étudié (réf. [7]) on se trouve dans le cas b du paragraphe 5 .

[13] Nomarski, G., Roblin, G. et Geleoc, P., C. R. Hebd. Séan. Acad. Sci. Paris 1969, 269, 290.

[14] Dillon, J. F., Magnetics properties of materials Inter University electronics series (1971) 149.

[15] Amzallag, E., Ann. Phys. 5 (1970) 27. 